\title{
ene
}

\section{Sistema de Alerta Temprana COVID-19 EN LA HISTORIA ELECTRÓNICA DE PACIENTES HOSPITALIZADOS}

\section{EARLY WARNING SYSTEM COVID-19 IN THE ELECTRONIC CARE RECORDS OF INPATIENTS}

Sandra Avellaneda-Martínez. RN1, Elena Barrios-Díez. RN², Francisco José Rodríguez-Alcázar. RN22, Natán Redondo-Pérez. MSc., RN1, Isaías del Río-García. MSc¹., RN, Belén Martín-Gil. RN11, Ángela Jiménez-Mayoral. RN1, Mercedes Fernández-Castro MSc., R.N1.

1 Hospital Clínico Universitario de Valladolid. España ${ }^{2}$ Servicio de Sistemas de Información y Resultados de Salud de la Gerencia Regional de Salud de Castilla y León, España. 


\section{Resumen}

Objetivo. Diseñar una alerta temprana con signos y síntomas de la enfermedad COVID-19 para detectar los distintos niveles de riesgo de padecer dicha enfermedad, establecer el nivel de vigilancia y activar los protocolos adecuados.

Método. Estudio descriptivo en tres fases: Revisión bibliográfica; Consenso entre expertos; Pilotaje de la alerta temprana

Resultados. Las variables predictoras de severidad para construir el algoritmo fueron: tos seca, fiebre, saturación de oxígeno, disnea y PCR. Se diseñó el algoritmo y se implementó en el aplicativo electrónico de gestión de Cuidados de Línea Abierta (GACELA Care)®.

Conclusiones. Con la implantación de la alerta temprana se espera una mejor gestión de los cuidados en los pacientes hospitalizados susceptibles de padecer COVI-19.

Palabras Clave: COVID-19; Alerta temprana; pacientes hospitalizados; registros electrónicos.
Abstract

Objective. To design an early warning system with signs and symptoms of COVID-19 disease in order to detect the levels of risk on suffering from this disease, to establish the level of vigilance and activate the appropriate protocols.

Method. A descriptive study in three phases: Bibliographic review; Consensus by experts and Piloting of early warning.

Results. Variables considered as predictors of severity to create the early warning algorithm were: dry cough, fever, oxygen saturation, dyspnea and PCR. The algorithm was designed and implemented in the Open Line Care Management electronic application (GACELA Care) ${ }^{\circledR}$.

Conclusions. With the implementation of early warning system, better care management is expected in inpatients that are susceptible to COVI-19.

Key words: COVID-19; Early warning system; inpatients, electronic record. 


\section{INTRODUCCIÓN}

El 31 de diciembre de 2019 las Autoridades de la República Popular China, comunicaron a la OMS varios casos de neumonía de etiología desconocida en Wuhan (China) (1). El 11 de febrero el virus causante fue nombrado SARS-CoV-2 por la Organización Mundial de la Salud (OMS), y la enfermedad asociada se denominó CoV Disease-19 (COVID-19) (2). El 12 de marzo la OMS, pasó a considerarlo de epidemia a pandemia (3). La proporción de la pandemia ha afectado a más de 100 países y aunque las medidas propuestas por los distintos gobiernos y por la OMS han reducido la aparición de nuevos casos en China y Europa, existe una gran preocupación con respecto a la capacidad de los sistemas de salud para responder a la necesidad de los pacientes infectados y especialmente aquellos que requieren cuidados intensivos $(4,5)$.

En España, desde el 14 de marzo, se ha decretado el estado de Alerta según Real Decreto 463/2020 por el rápido incremento de pacientes infectados con coronavirus del síndrome respiratorio agudo grave 2 (SARS-CoV-2) y fallecidos. Por ello se precisó la creación de grupos técnicos y profesionales para dar soporte a nuevas estrategias que permitan el abordaje y manejo de estos pacientes en los hospitales. Hasta el 14 de abril de 2020, en España se han confirmado 172.541 casos con 18.056 fallecidos (6). Diversos documentos técnicos del Ministerio de Sanidad han dado las pautas de actuación ante la pandemia, para la prevención y control de la infección en el manejo de pacientes con COVID-19 dentro de los hospitales (7). A partir de ellos la Gerencia Regional de Salud de Castilla y León, ha diseñado diferentes estrategias sobre procedimientos de actuación en el manejo del nuevo coronavirus (SARS-CoV-2), para poder dar soporte a la altísima incidencia de ingresos en las primeras semanas de la pandemia, dar la mejor calidad de atención y cuidados y proteger a los profesionales de la exposición al nuevo coronavirus. Por otro lado, los gestores dentro del hospital han adaptado todos sus recursos a las nuevas necesidades impuestas por la pandemia.

Según la OMS, un Sistema de Alerta Temprana se define como "un sistema integrado de vigilancia, previsión y predicción de amenazas, evaluación de los riesgos de desastres, actividades, sistemas y procesos de comunicación y preparación... que permite adoptar las medidas oportunas para reducir los riesgos de desastres con antelación a sucesos peligrosos"(8). Los Sistemas de Alerta Temprana en el ámbito sanitario se basan en la medición de diferentes pará- 
metros fisiológicos con el propósito de asegurar el reconocimiento oportuno de pacientes con enfermedades críticas potenciales o establecidas(9). Una vez detectados esos pacientes se procederá a la activación de un sistema de respuesta rápida que garantice las intervenciones adecuadas, evitando o disminuyendo las complicaciones derivadas de la patología detectada.

La nueva enfermedad, denominada COVID-19, presenta una rápida transmisibilidad a través del contacto con las secreciones respiratorias de una persona enferma, por gotas respiratorias de más de 5 micras (capaces de transmitirse a distancias de hasta 2 metros). La clínica varía desde un cuadro leve y de vías respiratorias altas, hasta un cuadro de neumonía grave con sepsis(10). La mayoría de las pruebas utilizadas actualmente para la detección directa de SARS-CoV-2 identifican el ARN viral a través de la amplificación de ácido nucleico, generalmente usando PCR (Reacción en Cadena de la Polimerasa). Los tipos de muestra más comunes que se analizan son los hisopos tomados de la nasofaringe y / o la orofaringe, siendo considerado el primero más sensible que el segundo. Tiene una especificidad y sensibilidad desconocida hasta el momento, aunque es importante reconocer que las pruebas basadas en ARN son las mejores pruebas existentes en el contexto de una enfermedad aguda (11).

Dada la funcionalidad de las alertas tempranas, los valores predictivos de los test y la herramienta electrónica de la que disponemos para la gestión de los cuidados, la utilización de un sistema de alerta temprana en el aplicativo electrónico de cuidados, de los pacientes hospitalizados, podría ser útil para establecer un mayor nivel de vigilancia por parte de las enfermeras, y realizar actuaciones que ayuden a gestionar los casos de pacientes que ingresan por otras causas ajenas a COVID-19, pacientes con sospecha de COVID-19 sin PCR o PCR negativa y pacientes con confirmación de la enfermedad mediante PCR positiva.

\section{Objetivo}

Diseñar una alerta temprana con signos y síntomas de la enfermedad COVID-19 que permitan detectar los distintos niveles de riesgo de padecer dicha enfermedad, en los pacientes hospitalizados, para establecer el nivel de vigilancia y activar los protocolos adecuados según los casos.

Implementar la alerta temprana en el aplicativo de cuidados que forma parte de la historia electrónica del paciente, Gestión de Cuidados de Línea abierta (GACELA Care) ${ }^{\circledR}$. 


\section{MÉTODO}

Estudio descriptivo llevado a cabo en tres fases:

Fase 1: Revisión bibliográfica con el fin de determinar la mejor evidencia disponible sobre los signos y síntomas determinantes en el diagnóstico clínico de la enfermedad producida por el virus SARS-CoV-2. La búsqueda se realizó en las bases de datos Medline, Cochrane library y CINHAL así como en los recursos electrónicos UpToDate, Publisher support for combating COVID-19, Novel Coronavirus Information Centre y Social Science Research Network en donde se publican estudios Pre-print. Todos los estudios consultados fueron publicados entre enero y marzo de 2020 y tenían entre sus objetivos profundizar en el conocimiento de cómo se comporta el coronavirus y las respuestas del organismo ante la infección. Las palabras de búsqueda fueron: (SARS-CoV-2); (COVID-19); Coronavirus disease 2019 (COVID-19); 2019-nCoV; Epidemic. La última búsqueda se realizó el 21 marzo de 2020.

Fase 2: Consenso entre expertos. Cinco expertos fueron consultados sobre la pertinencia de los síntomas y signos a considerar como variables determinantes para el diseño de la Alerta Temprana, así como la puntuación asig- nada al valor que debería tomar cada una de ellas (ver tabla 1), Tras la búsqueda bibliográfica, mediante una reunión presencial y trabajo online. Un médico especialista en Medicina Interna, un epidemiólogo, un neumólogo y dos enfermeras de la Unidad de Enfermedades Infecciosas.

Fase 3: Pilotaje de la alerta temprana. Desde el 23 al 27 de marzo se implementó solo para las plantas destinadas a pacientes con sospecha de COVID-19. Se reportaron incidencias por parte de las responsables del aplicativo de gestión de cuidados en el Hospital Río Carrión de Palencia y el Hospital Clínico Universitario de Valladolid y de alguna de las Jefes de Unidad de Enfermería en donde se puso en práctica, motivando cambios sobre el algoritmo original.

El diseño e implementación de la alerta temprana se llevó a cabo por Técnicos de Sacyl y del grupo Oesía® que dan soporte al aplicativo de Gestión de Cuidados de Línea Abierta (GACELA Care)®. Esta herramienta es la utilizada en todos los hospitales de la red Sacyl.

El ámbito de aplicación fue los 14 hospitales de la red de Salud de Castilla y León (Sacyl), que atienden a una población de 21320.373, según datos de la Población de Tarjeta sanitaria de castilla 
y León a fecha de 31 de diciembre de 2019 (12).

\section{REsultados}

Se revisaron 26 artículos de los cuales se extrajo que el espectro clínico de la infección por COVID-19 parece ser amplio, incluyendo infección asintomática, enfermedad leve de las vías respiratorias superiores, neumonía viral grave con insuficiencia respiratoria e incluso la muerte $(2,13,14)$. La mayoría de los pacientes que necesitaron hospitalización fue por desarrollar neumonía o un síndrome respiratorio agudo severo (15-17). Los síntomas comunes al inicio de la enfermedad fueron fiebre, tos seca, disnea y mialgia o fatiga $(14,15,17,18)$. Algunos estudios hallaron probabilidades crecientes de gravedad asociadas con la duración de la fiebre y presión parcial de oxígeno menor a 80 mmHG (18-20).

Por todo ello se consideraron como variables predictoras de gravedad para construir el algoritmo de la alerta temprana: tos seca, fiebre, saturación de oxígeno, disnea y PCR, para ser valoradas de manera sistemática por las enfermeras y permitir la detección de personas susceptibles de padecer la enfermedad.

Tras la búsqueda bibliográfica y el consenso entre expertos, las cinco variables descriptoras del algoritmo fue- ron puntuadas para un correcto reconocimiento de los distintos niveles de alerta, según la alteración con respecto a los valores establecidos como normales. La única variable con condición imprescindible para realizar el cálculo de nivel de alerta fue la temperatura, por su carácter predictor de mayor probabilidad de gravedad (Tabla1). Además, valoró si el paciente tiene test de PCR positiva, negativa o está pendiente de su realización.

La puntuación obtenida en la valoración del paciente se asoció a una codificación en colores, además de proponer una recomendación específica a ese nivel de puntuación (ver tabla 2).

Nivel 1. Color Gris. Puntuación del algoritmo entre 1 y 3 . Recomendación específica: periodicidad de toma de variables según pauta habitual.

Nivel 2. Color Amarillo. Puntuación del algoritmo entre 4 y 7 . Recomendación específica: aumento de la periodicidad de la toma de variables cada 8 horas.

Nivel 3. Color Naranja. Puntuación del algoritmo entre 8 y 12 . Recomendación específica: comunicación al médico responsable para valoración de nueva prueba PCR y valoración de aislamiento preventivo.

Nivel 4. Color Rojo. Puntuación del algoritmo $\geq 13$ o simplemente PCR 
positiva (valor 5) considerada ésta última como Parámetro Individual. En este caso, aunque la PCR positiva no vaya acompañado de la suma de otras variables, el paciente pasa automáticamente a nivel 4. Recomendación específica: Aislamiento por confirmación de COVID19 positivo, según protocolo de la unidad.

Para implementar el algoritmo se ha utilizado el módulo de alerta temprana del aplicativo GACELA Care®. Este módulo tiene cuatro servicios de configuración del algoritmo:

Algoritmos de puntuación: Diseño de la puntuación que adjudicas a cada una de cada una de las variables dependiendo del valor que presentan cada una de ellas.

Estratificación y respuesta: Genera niveles de alerta y de respuesta según qué puntuación global adquieren las distintas variables utilizadas, donde cada uno de ellos cambia de color el icono del paciente. En este apartado, se puede añadir la condición de un paciente con la característica de COVID-19 positivo confirmado y generar un nivel específico.

Destinos de llamada: Si fuera necesario, indica a quien hay que notificar el nivel de alerta adquirido por el paciente.

Modelos de alerta temprana: Almacena en único valor los distintos algo- ritmos y estratificaciones que se han generado.

El registro del nivel de alerta temprana puede ser generado directamente en los comentarios evolutivos del paciente e informa a la enfermera si es necesario notificar o realizar alguna acción concreta según el nivel de alerta.

Una vez implementada la alerta temprana se informó de la disponibilidad de la herramienta a las Direcciones de Enfermería y a las responsables de los sistemas de información de los 14 hospitales de Sacyl. En la actualidad en cada hospital se está implantado la alerta temprana en aquellas unidades donde se ha considerado de mayor utilidad, cambiando según las necesidades de adaptabilidad en la gestión de las distintas unidades de hospitalización.

\section{DisCUSIÓN / IMPLICACIONES PARA LA PRÁCTICA}

La aplicación de una alerta temprana podría ser oportuna en este caso en el que nos enfrentamos a un patógeno con niveles elevados de morbimortalidad e implicaciones graves en salud pública. Este tipo de herramientas refuerza otras medidas de prevención de riesgos y exposición a agentes infecciosos de los distintos profesionales que 
trabajan en la mejora de la salud de los pacientes.

La principal ventaja de este sistema de alerta temprana es que utiliza la mayor parte de variables clínicas que frecuentemente manejan y registran las enfermeras. Este hecho se comprobó en los primeros días, donde el despliegue de esta alerta temprana fue asociado al registro de estas variables clínicas y a un incremento en las de nueva creación como fue la presencia de tos.

La aplicación de esta medida ha sido de una forma abrupta y condicionada a las necesidades diarias de los distintos servicios de hospitalización. Esto podría generar que su despliegue se realice de forma sistemática convirtiéndolo en algo rutinario perdiendo el valor añadido que se pretende dar al trabajo asistencial.

\section{CONCLUSIONES}

Con la implantación de la alerta temprana se espera lograr una mejor gestión de los cuidados de los pacientes hospitalizados mientras exista el riesgo de enfermedad COVID-19.

A raíz de la implantación y la generalización de la alerta temprana en los hospitales de Sacyl, se están diseñando nuevos estudios para analizar: el grado de adherencia en la cumplimentación de las variables que generan la alerta (tos seca, fiebre, saturación de oxígeno, disnea y PCR), la capacidad real de la herramienta para predecir el riesgo, la utilidad y la efectividad de las actuaciones según los niveles de alerta.

\section{FINANCIACIÓN}

Ninguna.

\section{AGRADECIMIENTOS}

Nuestro agradecimiento a los Técnicos del grupo Oesía, gestores del aplicativo de Gestión de Cuidados de Línea Abierta (GACELA Care)®.

\section{Consideraciones ÉTICAS}

Este trabajo se ajusta a la Ley Orgánica 3/2018, de 5 de diciembre, de Protección de Datos Personales y garantía de los derechos digitales. No precisó aprobación de Comité Ético alguno.

\section{CONFLICTO DE INTERESES}

Los autores declaran no tener conflicto de intereses. 


\section{BiBLIOGRAFÍA}

Velavan TP, Meyer CG. (2020) The COVID-19 epidemic. Trop Med Int Health; 25: 278-80. DOI: 10.1111/tmi.13383

2. Yi Y, Lagniton PNP, Ye S, Li E, Xu R-H. (2020) COVID-19: what has been learned and to be learned about the novel coronavirus disease. Int J Biol Sci;16(10): 1753-66. DOI: 10.7150/ijbs. 45134

3. Organización Mundial de la Salud (OMS). (2020) Coronavirus disease 2019. Novel coronavirus (2019-nCoV) situation report. Consultado 15 Abril, 2020, de la Wold Wide Web: https://www.who.int/emergencies/diseases/novelcoronavirus-2019

4. Kandel N, Chungong S, Omaar A, Xing J. (2020) Health security capacities in the context of COVID-19 outbreak: an analysis of International Health Regulations annual report data from 182 countries. Lancet. 2020;395: (1047-53). Consultado 15 Abril, 2020, de la Wold Wide Web: http:// dx.doi.org/10.1016/S0140-6736(20)30553-5

5. Lazzerini M, Putoto G (2020) COVID-19 in Italy: momentous decisions and many uncertainties. Lancet Glob Heal. Consultado 15 Abril, 2020, de la Wold Wide Web: http://dx.doi.org/10.1016/ S2214-109X(20)30110-8

6. Ministerio de Sanidad, Consumo y Bienestar Social. Enfermedad por nuevo coronavirus, COVID-19. (2020) Madrid: Ministerio de Sanidad, Consumo y Bienestar Social. Consultado 15 Abril, 2020, de la Wold Wide Web: https://www.mscbs.gob.es/profesionales/saludPublica/ccayes/alertasActual/nCov-China/home.htm

7. Ministerio de sanidad. Documento técnico Prevención y Control de la infeccion en el manejo de pacientes con COVID-19 (2020) Madrid. Ministerio de Sanidad. Consultado 15 de abril, 2020, de la Wold Wide Web: https://www.mscbs.gob.es/ profesionales/saludPublica/ccayes/alertasActual/ nCov-China/documentos/Documento_Control_Infeccion.pdf

8. Organización Panamericana de la Salud. Lista de verificación de alistamiento para la respuesta al COVID-19 en Hospitales (2020, Febrero) Consultado el 15 de abril, 2020, de la Wold Wide Web: https://www.paho.org/hq/index.php? option=com_docman\&view=download\&alias $=5171$ 8-lista-de-verificacion-de-alistamiento-para-la-respuesta-al-2019-ncov-en-hospitales\&category_sI u $\mathrm{g}=\mathrm{m}$ a t e ri a le s - cientificos tecnicos-7992\&ltemid=270\&lang=es

9. Jensen JK, Skår R, Tveit B. (2018) The impact of Early Warning Score and Rapid Response Systems on nurses' competence: An integrative literature review and synthesis. J Clin Nurs, 27: 1256-74. DOI: 10.1111/jocn.14239
10. Guo YR, Cao QD, Hong ZS, Tan YY, Chen $\mathrm{SD}$, Jin HJ, et al. (2019) The origin, transmission and clinical therapies on coronavirus disease 2019 (COVID-19) outbreak - an update on the status. Mil Med Res, 7: 11. DOI: 10.1186/s40779-020-002400

11. Patel R, Babady E, Theel ES, Storch GA, Pinsky BA, St George K, et al. (2020) Report from the American Society for Microbiology COVID-19 International Summit, 23 March 2020: Value of Diagnostic Testing for SARS-CoV-2/COVID-19. mBio, 11(2): e00722-20. DOI: 10.1128/mBio. 00722-20

12. Población de tarjeta sanitaria de Castilla y León. Diciembre 2019. (2019, diciembre). Secretaria General de Sanidad Y Consumo. Consultado 13 abril, 2020, de la Wold Wide Web: https://n9.cl/ ur1f

13. Ahn D-G, Shin H-J, Kim M-H, Lee S, Kim HS, Myoung J, et al. (2020) Current Status of Epidemiology, Diagnosis, Therapeutics, and Vaccines for Novel Coronavirus Disease 2019 (COVID-19). J Microbiol Biotechnol, 30(3): 313-324. Consultado 13 abril, 2020, de la Wold Wide Web: http:// www.ncbi.nlm.nih.gov/pubmed/32238757

14. Adhikari SP, Meng S, Wu YJ, Mao YP, Ye RX, Wang QZ, et al.(2020) Epidemiology, causes, clinical manifestation and diagnosis, prevention and control of coronavirus disease (COVID-19) during the early outbreak period: A scoping review. Infect Dis Poverty, 9(1): 29. DOI: 10.1186/ s40249-020-00646-x.

15. Gao J, Tian Z, Yang X. (2020) Breakthrough: Chloroquine phosphate has shown apparent efficacy in treatment of COVID-19 associated pneumonia in clinical studies. Biosci Trends, 14(1): 72-3. DOI: 10.5582/bst.2020.01047

16. Lake MA. (2020) What we know so far: COVID-19 current clinical knowledge and research. Clin Med, 20(2): 124-7. DOI: 10.7861/ clinmed.2019-coron

17. Jin Y, Yang H, Ji W, Wu W, Chen S, Zhang W, et al. (2020) Virology, Epidemiology, Pathogenesis, and Control of COVID-19. Viruses, 87(4): 281-6. Consultado 15 abril, 2020, de la Wold Wide Web: https://www.mdpi.com/ 1999-4915/12/4/372

18. Ryu S, Chun BC; Korean Society of Epidemiology 2019-nCoV Task Force Team. An interim review of the epidemiological characteristics of 2019 novel coronavirus. Epidemiol Health. 2020;42:e2020006. doi:10.4178/epih.e2020006

19. Docherty $A B$, Harrison EM, Green $C A$, et al. Features of 20133 UK patients in hospital with covid-19 using the ISARIC WHO Clinical Characterisation Protocol: prospective observational cohort study. BMJ 2020;369:m1985-m1985. DOI: 10.1136/bmj.m1985 
20. Wynants L, Van Calster B. Bonten MM.J., Collins, GS, Debray TPA, De Vos M, et al. (2020). Prediction models for diagnosis and prognosis of COVID-19 infection: Systematic review and critical appraisal. The BMJ, 369:m1328. DOI: 10.1136/ bmj.m1328.

\section{TABLAS Y ANEXOS}

Tabla 1. Puntuación de las variables clínicas incluidas en la alerta establecida por consenso de expertos.

\begin{tabular}{|c|cc}
\hline Variables Clínicas & Rango & Puntuación \\
\hline \multirow{2}{*}{ Disnea } & NO & 1 \\
\hline \multirow{2}{*}{ PCR } & SI & 3 \\
\hline \multirow{2}{*}{ Saturación oxígeno } & + & 5 \\
\hline \multirow{2}{*}{$\begin{array}{c}\text { Temperatura } \\
\text { (obligatoria) }\end{array}$} & No & 0 \\
\hline \multirow{2}{*}{} & $\leq 90$ & 3 \\
\hline Tos & $35.0-37,4^{\circ} \mathrm{C}$ & 0 \\
\hline & $37,5-38,5^{\circ} \mathrm{C}$ & 1 \\
\hline & $\geq 38,5$ & 3 \\
\hline & No & 4 \\
\hline & Productiva & 0 \\
\hline & Seca & 1 \\
\hline
\end{tabular}


Tabla 2. Configuración de la alerta temprana con la puntuación de los niveles, actuaciones y colores.

\begin{tabular}{|c|c|c|c|c|}
\hline Nivel & Condición & $\begin{array}{l}\text { Características } \\
\text { Paciente }\end{array}$ & Actuación & Icono \\
\hline \multirow[t]{2}{*}{$\begin{array}{l}\text { Nivel 1- } \\
\text { Seguimiento }\end{array}$} & \multirow[t]{2}{*}{$\begin{array}{l}\mathrm{PTA}^{*}: 0-3 \mathrm{PPI}^{\star \star}: \\
0-3\end{array}$} & Cualquiera & $\begin{array}{l}\text { Toma de variables según } \\
\text { pauta habitual }\end{array}$ & \\
\hline & & COVID19:PCR+ & Aislamiento & \\
\hline \multirow[t]{2}{*}{$\begin{array}{l}\text { Nivel } 2 \text { - } \\
\text { Vigilancia }\end{array}$} & \multirow[t]{2}{*}{ PTA* $^{\star}: 4-7$} & Cualquiera & $\begin{array}{l}\text { Toma de variables cada } \\
\text { 8horas }\end{array}$ & \\
\hline & & COVID19:PCR+ & Aislamiento & \\
\hline \multirow[t]{2}{*}{ Nivel 3 - Riesgo } & \multirow[t]{2}{*}{$\mathrm{PTA}^{*}: 8-12$} & Cualquiera & $\begin{array}{l}\text { PCR si precisa y } \\
\text { aislamiento preventivo }\end{array}$ & \\
\hline & & COVID19:PCR+ & Aislamiento & \\
\hline \multirow[t]{2}{*}{$\begin{array}{l}\text { Nivel } 4- \\
\text { Aislamiento }\end{array}$} & \multirow[t]{2}{*}{$\begin{array}{l}\mathrm{PTA}^{*} \geq 13 \\
\mathrm{PPI}^{*} \geq 5\end{array}$} & Cualquiera & Aislamiento & \\
\hline & & COVID19:PCR+ & Aislamiento & \\
\hline
\end{tabular}

*PTA: Puntuación Total del Algoritmo. **PPI: Puntuación Parámetro Individual 